\title{
Terapia fotodinâmica: revisão de literatura
}

\section{Photodynamic therapy: a review}

\section{Resumo}

Dentre as modalidades terapêuticas recentemente investigadas na comunidade científica, destaca-se a terapia fotodinâmica. Sua aplicação vem sendo empregada na destruição de células tumorais e na inativação de microorganismos, com grande perspectiva em diversas áreas da Medicina, Odontologia e, mais recentemente, na Medicina Veterinária. O presente estudo tem como objetivos abordar trabalhos na literatura mundial e informar médicos veterinários sobre a terapia fotodinâmica e suas possíveis aplicações na Medicina Veterinária.

\section{Summary}

Among the most recent therapeutic modalities investigated in the scientific community, stands the photodynamic therapy. It has been used for destruction of tumor cells and microorganisms inactivation, with great prospects in different fields of Medicine, Dentistry and most recently in Veterinary Medicine. The purposes of this review are to address the world literature and inform veterinarians about photodynamic therapy and its possible applications in veterinary medicine. 
Fábio Parra Sellera ${ }^{1}$

Ronaldo Gomes Gargano ${ }^{1}$

Fabio Celidonio Pogliani ${ }^{1}$ 的ellera@usp.br

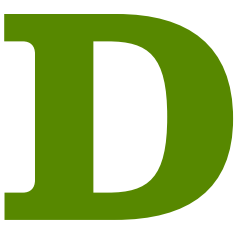

esde os tempos primitivos, o homem busca alternativas para combater as diversas enfermidades que desafiam a vida. A história da terapia fotodinâmica moderna remonta às tentativas de antigos egípcios para tratar doenças da pele com compostos absorvíveis pela luz. Documentos datados com seis mil anos de idade relatam que os antigos egípcios aplicavam substâncias vegetais para produzir fotorreações nos tecidos. No Egito, Índia e China, a luz solar era utilizada para tratamento de doenças da pele como psoríase, vitiligo e câncer, descritos a partir do papiro de Ebers (WEISS et al., 2012) e do livro sagrado indiano Atharva Veda (WYSS, 2000). $\mathrm{Na}$ Grécia antiga, o médico grego Heródoto definia a técnica como Helioterapia e ressaltava a importância da exposição à luz solar para a restauração da saúde (ACKROYD et al., 2001).

Em 1900, relatou-se a primeira descrição científica de tratamento fotodinâmico, realizado por um estudante de medicina, Oscar Raab, e por seu professor, Herman Von Tappeiner, em Munique. Esses pesquisadores investigavam o efeito do corante de acridina sobre culturas de paramécios e observaram que a interação desse corante com a luz foi letal para os protozoários. Durante uma tempestade e exposição a muitos raios, houve alteração das condições luminosas do ambiente no momento dos experimentos, direcionando os autores a postular que o efeito observado fosse causado pela transferência da energia da luz para 
a substância química acridina; fato similar ao que ocorre nas plantas pela absorção da luz pela clorofila. A ação fotodinâmica foi comprovada quando se observou que a luz e o corante, isoladamente, não apresentaram qualquer efeito sobre os protozoários (ACKROYD et al., 2001).

Em 1901, Niels Finsen publicou o primeiro livro voltado para o tratamento de doenças através da luz, intitulado "Phototherapy" e, dois anos após, em 1903, o mesmo pesquisador foi premiado com o Prêmio Nobel na área de Medicina, pelo uso da eosina e da luz no tratamento de lupus vulgaris (PENG; MOAN; NESLAND, 1996; ALLISON et al., 2004).

Em 1901, Hereon Henrian Von Tappeiner descobriu que o oxigênio era necessário para o desenvolvimento da resposta das reações mediadas pela luz. Assim foi criado o termo "terapia fotodinâmica", conhecido até os dias atuais, para descrever essa terapia oxigênio-dependente (PENG; MOAN; NESLAND, 1996; ALLISON et al., 2004; BABILAS et al., 2005). Ainda no mesmo ano, Von Tappeiner e Jesionek investigaram a aplicação de eosina e luz para tratar tumores de pele (DOUGHERTY et al., 1978; KESSEL, 2004).

A terapia fotodinâmica passou por um grande avanço em 1908, quando surgiram os primeiros relatos sobre o uso de porfirinas como substâncias fotossensibilizadoras. Após dezesseis anos, em 1924, Policard detectou fluorescência de tumores expostos à porfirina e em seguida irradiados com lâmpada de Wood; fato que enalteceu as pesquisas efetuadas por volta do final da década de 40, quando foi descoberto que as porfirinas se acumulavam preferencialmente em tumores (ACKROYD et al., 2001).

Em 1916, Albert Einsten postulou a primeira teoria sobre laser, "os princípios da luz pela emissão estimulada de radiação", que posteriormente baseou o desenvolvimento do primeiro laser, em 1960, por Theodore Maiman, desencadeando uma série de pesquisas envolvendo a interação entre luz e tecido (FELDMAN, 2009).

$\mathrm{Na}$ década de 70, a Medicina ganhou um forte aliado na guerra contra o câncer, quando Thomas Dougherty deu início a uma série de pesquisas clínicas envolvendo os derivados da hematoporfirina, desenvolvendo a formulação dessas substâncias em larga escala, segundo as normas estabelecidas pela Food and Drug Administration (FDA) (KESSEL, 2004). A partir da mesma década, relatos dos efeitos da resistência microbiana aos antibióticos, observados anos após a descoberta da penicilina por Alexandre Fleming, em 1928, incentivaram e desencadearam uma série de pesquisas que continuam a ser realizadas nos dias atuais, envolvendo a terapia fotodinâmica no controle de microorganismos.

\section{Princípios}

A terapia fotodinâmica, mais comumente denominada PDT, do inglês "Photodynamic Therapy", é uma das modalidades terapêuticas mais estudadas na área da saúde nas últimas décadas. Sua aplicação envolve o uso de um agente fotossensibilizador, luz e oxigênio molecular para destruir tanto células neoplásicas (ZEITOUNI; OSEROFF; SHIEH, 2003; MARMUR; SCHMULTS; GOLDBERG, 2004) como também microorganismos (bactérias, vírus e fungos). Não apresenta efeitos genotóxicos e mutagênicos e, portando, não possibilita o desenvolvimento de resistência microbiana (KONOPKA; GOSLINSKI, 2007).

O efeito fotodinâmico da PDT ocorre quando a molécula do fotossensibilizador absorve fótons, emitidos pela fonte de luz de comprimento de onda específico e ressonante ao fotossensibilizador, passando de seu estado fundamental para um estado excitado. Nesse momento pode ocorrer a transformação de energia por processos de fluorescência ou conversão interna do estado excitado para o estado fundamental e/ou ocorrer o processo de cruzamento entre sistemas, levando a molécula para o estado excitado tripleto. Nesse estado, as moléculas do fotossensibilizador podem interagir com o meio através de dois processos distintos, denominados de mecanismos tipo I e tipo II. No mecanismo tipo I, as reações de transferência de elétrons ocorrem entre o fotossensibilizador e o substrato, formando espécies de radicais livres. No mecanismo tipo II, ocorre transferência de energia do fotossensibilizador ao oxigênio no estado fundamental, havendo a formação de oxigênio singleto. Os fatores que determinarão a morte seletiva de células do hospedeiro ou a inativação microbiana estão diretamente relacionados com a molécula fotossensibilizante empregada e as dosimetrias química e luminosa (ALLISON et al., 2004; HAMBLIN; HASAN, 2004).

\section{Indicações}

A PDT pode ser indicada no tratamento de uma grande variedade de neoplasias cutâneas em cães e gatos. Além disso, o seu uso também foi investigado em neoplasias localizadas em cavidade oral, esôfago, próstata e cérebro, em animais e seres humanos. A aplicação da PDT em neoplasias malignas de origem não epitelial, como glioma, retinoblastoma, lipossarcoma e condrossarcoma, também é descrita na literatura (LUCROY, 2002; EMILIO, 2008).

Apesar de estudos envolvendo animais serem apontados desde a década de 80 , protocolos terapêuticos na veterinária ainda são amplamente discutidos, devido ao fato de existirem muitas variáveis nesse tipo de procedimento e poucos trabalhos na literatura quando comparado a outras áreas da saúde, como Medicina e Odontologia. 

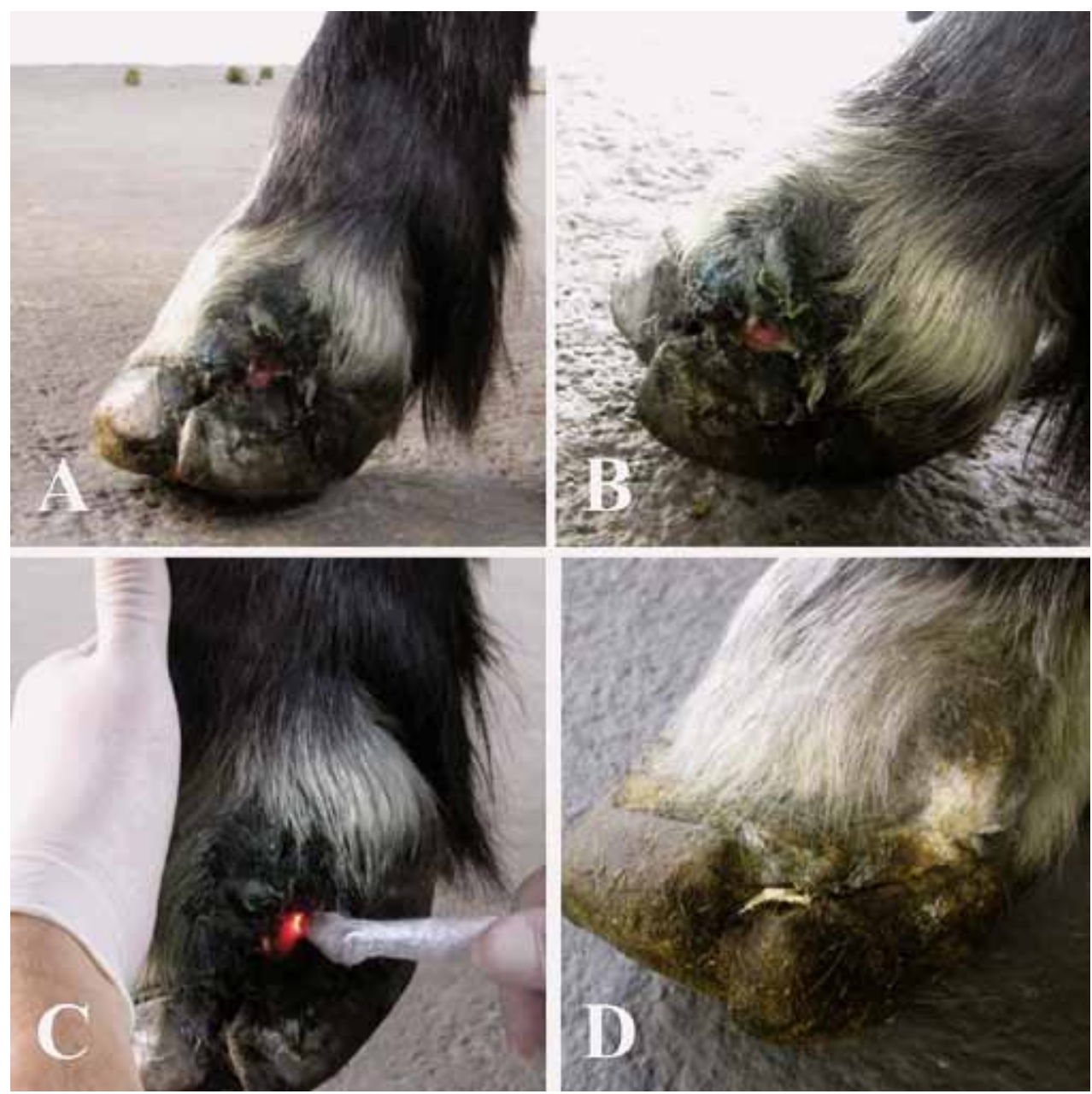

Figura 1 - Imagens fotográficas da evolução de uma ferida no casco de um equino tratado com a terapia fotodinâmica. (A e B) Imagens da lesão antes do tratamento; (C) Aplicação da PDT; (D) Aspecto da lesão após uma semana
Além disto, a crescente preocupação em relação à seleção de micro-organismos resistentes aos antibióticos vem sendo discutida incessantemente, uma vez que as infecções causadas por diversos patógenos oportunistas em animais crescem a cada ano (MATEU; MARTIN, 2001). O emprego dessa técnica em infecções locais, indiscutivelmente, chama a atenção de médicos veterinários dermatologistas. Inúmeros patógenos (bactérias, fungos e vírus) revelaram-se susceptíveis in vitro (HAMBLIN; HASAN, 2004), o que torna a sua aplicação in vivo uma opção terapêutica a ser explorada.

Além do mais, a utilização da PDT no tratamento de feridas tem se mostrado muito promissora. As feridas tratadas exclusivamente com PDT apresentaram cicatrização mais rápida, quando comparadas ao tratamento usual. Admite-se que possa haver um efeito sinérgico entre os efeitos da luz e o fotossensibilizador, além do efeito antimicrobiano determinado pela PDT ser um adjuvante na reparação tecidual, principalmente por diminuir a carga microbiana (SILVA et al., 2004; SELLERA et al., 2013c).

\section{Principais agentes fotossensibilizadores}

Os principais agentes fotossensibilizadores e suas respectivas bandas de absorção de luz são apresentados na tabela 1.

Tabela 1 - Principais agentes fotossensibilizadores e suas respectivas bandas de absorção de luz

\begin{tabular}{lc}
\hline Agentes fotossensibilizadores & Banda de absorção \\
\hline Acridina & $400-500 \mathrm{~nm}$ \\
\hline Cianinas & $600-805 \mathrm{~nm}$ \\
\hline Derivados da porfirina (Photofrin, ALA) & $620-650 \mathrm{~nm}$ \\
\hline Fenotiazinas (Azul de toluidina, Azul de metileno) & $620-700 \mathrm{~nm}$ \\
\hline Fitoterápicos (Azuleno) & $550-700 \mathrm{~nm}$ \\
\hline Ftalocianinas & $660-700 \mathrm{~nm}$ \\
\hline Triarilmetano & $617-630 \mathrm{~nm}$
\end{tabular}

Fonte: adaptado de (ACKROYD, 2001; USACHEVA et al., 2001; LUCROY, 2002)

\section{Fontes de luz}

As primeiras fontes de luz empregadas na PDT foram as lâmpadas convencionais, com luz não coerente, policromática, e forte componente térmico, resultando em 

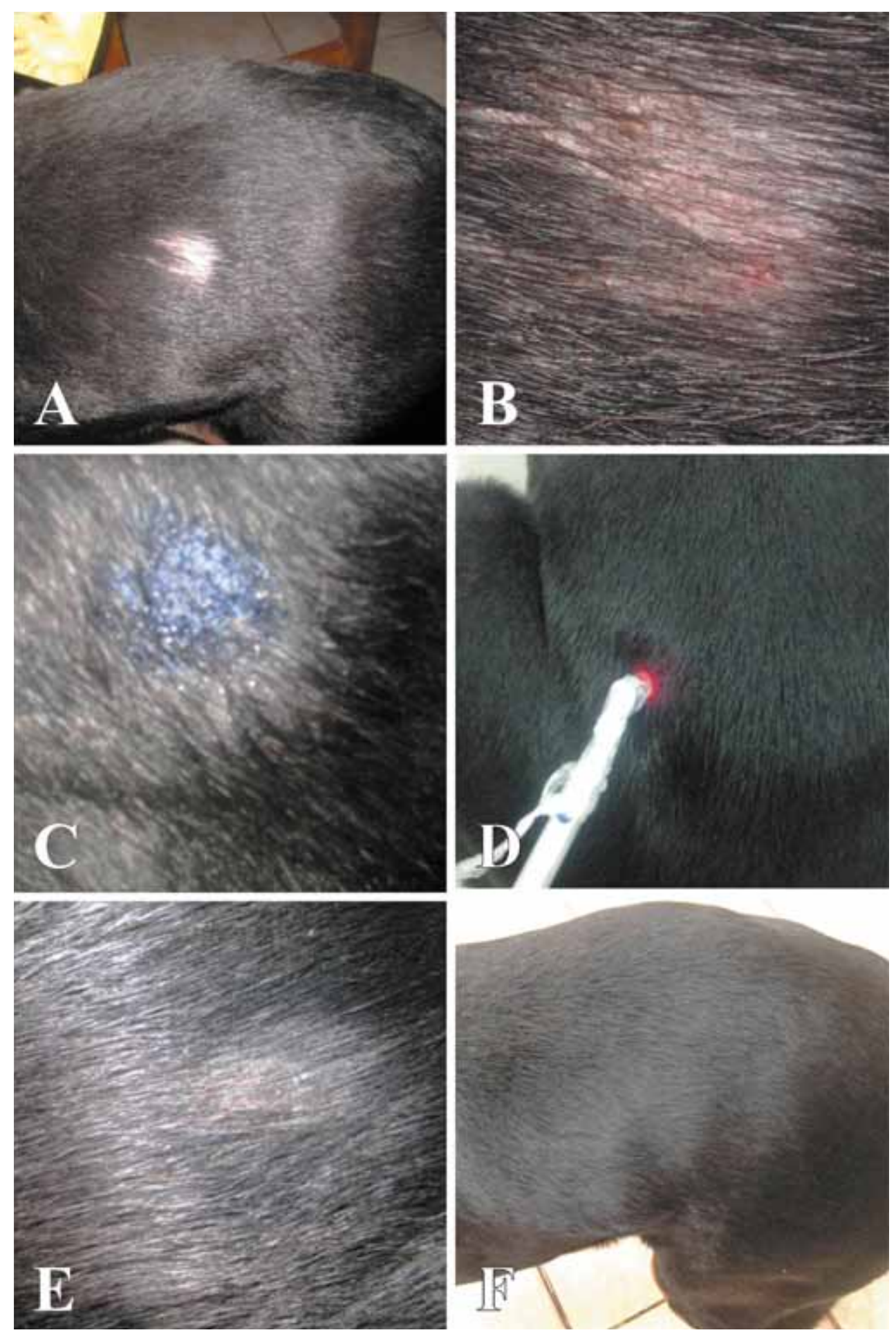

Figura 2 - Imagens fotográficas da evolução de uma dermatofitose na região dorsal em um cachorro tratado com a terapia fotodinâmica. (A e B) Imagens da lesão antes do tratamento; (C) Aplicação do agente fotossensibilizador (Azul de metiledo) (D) Aplicação da PDT (E) Lesão após uma semana de terapia (F) Aspecto do animal após duas semanas

aumento de temperatura da área irradiada. O desenvolvimento do laser possibilitou o emprego de uma fonte de luz de baixa intensidade, capaz de estimular a reação fotodinâmica (ACKROYD et al., 2001).

Os lasers emitem luz monocromática, com um comprimento de luz conhecido, o que os torna altamente seletivos aos fotossensibilizadores. A dose de radiação pode ser facilmente calculada, a área a ser tratada pode ser bem controlada e, além disto, a radiação pode ser transmitida por fibra óptica, o que facilita a entrega de luz em determinadas circunstâncias (ACKROYD et al., 2001).

Dentre os lasers desenvolvidos ao longo dos anos, os de diodo vêm se destacando por possuírem comprimento de onda ressonante à banda de absorção da 


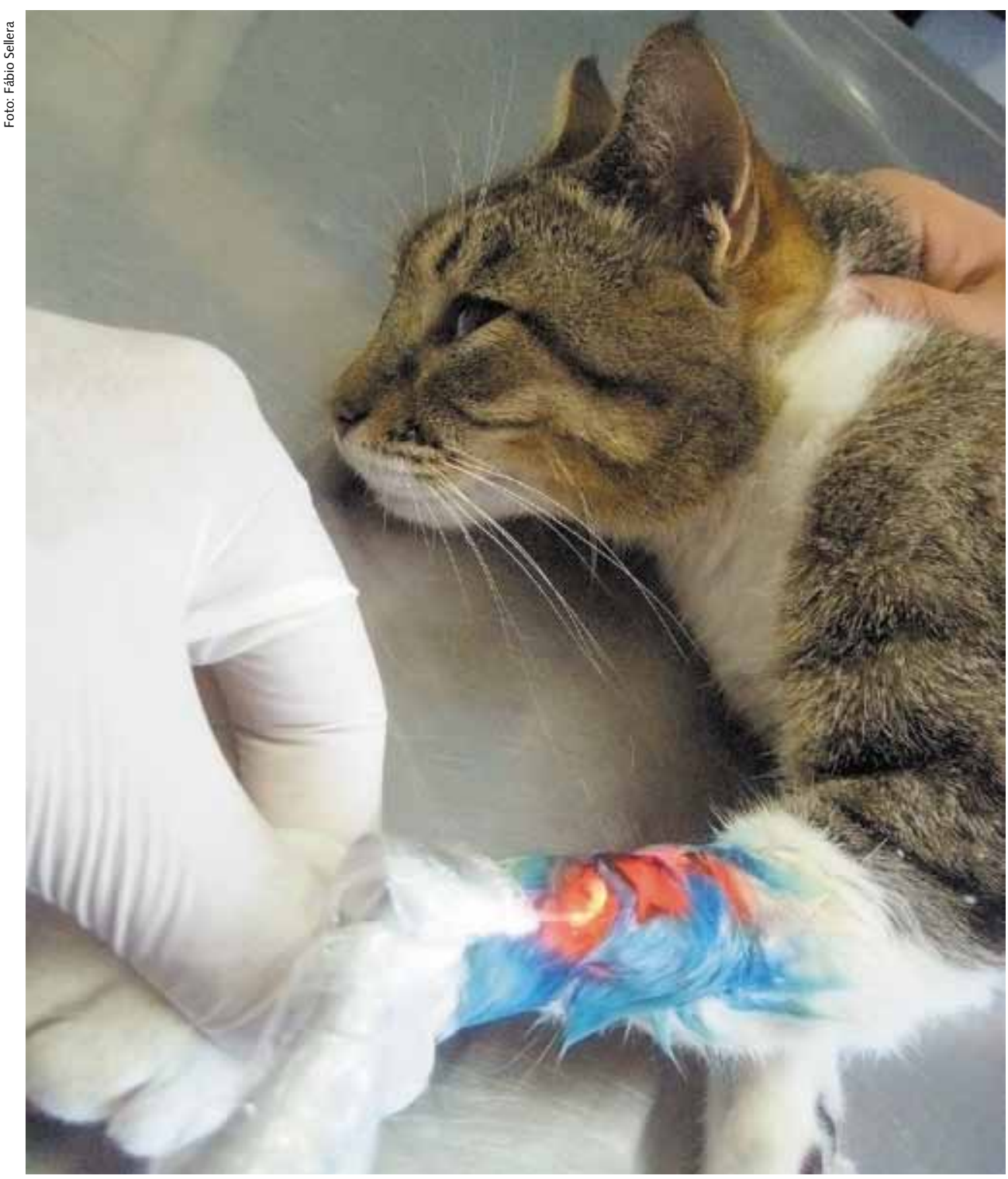

Figura 3 - Ferida infectada de felino é tratada com Terapia Fotodinâmica (PDT) maioria dos fotossensibilizadores atualmente utilizados; são menores, portáteis e de baixo custo; além de apresentarem grande poder de penetração nos tecidos biológicos (TAKASAKI et al., 2009).

Mais recentemente, os equipamentos constituídos por LEDs ("Light Emitting Diodes" - diodos emissores de luz) estão ganhando espaço como fontes de luz para a PDT, principalmente por serem monocromáticos, apresentarem baixo custo, reduzido componente térmico e promoverem a irradiação de superfície ampla (CALZAVARA-PINTON; VENTURINI; SALA, 2007).

\section{Efeitos colaterais}

O surgimento de efeitos colaterais está diretamente ligado às características químicas e concentração do fotossensibilizador, dosimetria luminosa empregada e tecido alvo.
No tratamento de infecções, aparentemente poucos ou nenhum efeito colateral são descritos. Isso se deve ao fato de a maioria dos fotossensibilizadores empregados para esse tipo de tratamento serem mais tóxicos aos microorganismos quando comparados às células dos hospedeiros (HAMBLIN; HASAN, 2004).

Os efeitos colaterais geralmente são observados nos casos de tratamento dos pacientes oncológicos, sendo descritos principalmente casos de fotossensibilização cutânea e efeitos indesejáveis como náusea, vômito e alterações transitórias de enzimas hepáticas em pacientes que receberam administração sistêmica de alguns fotossensibilizadores. Além disso, o tratamento tópico com PDT também pode desencadear sensações dolorosas, ardência ou prurido restrito à área irradiada (ZELICKSON, 2005; EMILIO, 2008). 


\section{Perspectiva na Medicina Veterinária}

A investigação sobre o uso da PDT em Medicina Veterinária iniciou-se na década de 80 , quando cães e gatos eram tratados com derivados de hematoporfirina e em seguida os tumores foram irradiados com laser. Embora as pesquisas envolvessem um número limitado de animais e uma grande variedade de tipos tumorais, grande parte dos tumores respondeu aos protocolos estabelecidos (LUCROY, 2002; EMILIO, 2008). Em 2007, Hage et al. relataram a aplicação intratumoral do ácido 5-aminolevulínico (ALA), substância precursora de protoporfirina IX, um agente fotossensibilizante, em carcinoma espinocelular de bovinos, e, mais recentemente, Emilio (2008) comparou a eficácia do ALA e de seu metil (MEALA) em gatos que apresentavam carcinoma espinocelular, indicando uma nova possibilidade terapêutica para a doença.

A PDT foi eficaz no tratamento de animais marinhos em reabilitação. Em pinguins de Magalhães (Spheniscus magellanicus) com podermatite, a PDT acelerou o processo de reparação tecidual e mostrou-se como uma alternativa para as opções convencionais de tratamento da enfermidade (SELLERA et al., 2012a). O tratamento de fibropapilomatose cutânea em tartarugas-verdes (Chelonia mydas) também foi relatado com alternativa viável para a doença (SELLERA et al., 2012b). O seu uso no tratamento de diferentes enfermidades podais dos bovinos e pequenos ruminantes tem demonstrado que a utilização dessa técnica permite melhor recuperação e em menor tempo que os respectivos tratamentos convencionais (SELLERA et al., 2012a,c).

A efetividade da PDT foi demonstrada no controle da presença de patógenos em sêmen bovino (EAGLESOME et al., 1994), no tratamento in vitro e in vivo de sarcóide em equinos (MARTENS et al., 2000), na inativação do vírus da artrite encefalite caprina no colostro (WASHBURN et al., 2001), no tratamento de tumor venéreo transmissível (TVT) em cães (VILENSKY et al., 2005), na desinfecção de água e tratamento de patógenos causadores de enfermidades em peixes e crustáceos (JORI et al., 2011) e no tratamento in vitro e in vivo da pitiose equina (PIRES et al., 2013).

Nas últimas décadas, a PDT está em evidência na Medicina Veterinária (LUCROY, 2002; EMILIO, 2008; OSAKI et al., 2012; SELLERA et al., 2013b). A sua aplicação no controle de microorganismos resistentes (HAMBLIN; HASAN, 2004) e na terapia de neoplasias superficiais (LUCROY, 2002; EMILIO, 2008) certamente a tornará essencial para os Médicos Veterinários (EMILIO, 2008).

\section{Considerações Finais}

O levantamento bibliográfico realizado confirma que, devido aos resultados promissores e à simplicidade da técnica, a PDT pode ser uma alternativa eficaz para o tratamento de diferentes enfermidades. Contudo, novas pesquisas são fundamentais para o desenvolvimento e elucidação da técnica e, além disso, para o desenvolvimento de protocolos terapêuticos, para que a técnica seja implementada de maneira eficiente e segura na rotina dos Médicos Veterinários. (-)

\section{Referências}

1. ACKROYD, R.; KELTY, C.; BROWN, N.; REED, M. The history of photodetection and photodynamic therapy. Photochemistry and Photobiology, v. 74, n. 5, p. 656-669, 2001.

2. ALLISON, R. R.; DOWNIE, G. H.; CUENCA, R.; HU, X. H.; CHILDS, C. J. H.; SIABATA, H. C. Photosensitizers in clinical PDT. Photodiagnosis and Photodynamic Therapy, v. 1, n. 1, p. $27-42,2004$

3. BABILAS, P.; KARRER, S.; SIDOROFF, A.; LANDTHALER, M.; SZEIMIES, R. $M$. Photodynamic therapy in dermatology - an update. Photodermatology, Photoimmunology \& Photomedicine, v. 21, p.142-149, 2005.

4. CALZAVARA-PINTON, P. G.; Venturini, M.; SALA, R. Photodynamic therapy: update 2006. Part 1: Photochemistry and photobiology. Journal of the European Academy of Dermatology and Venereology, v. 21, n. 3, p. 293-302, 2007.

5. DOUGHERTY, T. J.; KAUFMAN, J. E.; GOLDFARB, A.; WEISHAUPT, K. R.; BOYLE, $D$. Photoradiation therapy for the treatment of malignant tumors. Cancer Research, v. 38, p. $2628-2635,1978$.

6. EAGLESOME, M. D.; BIELANSKI, A.; HARE, W. C. D.; RUHNKE, H. L. Studies on inactivation of pathogenic microorganisms in culture media and bovine semen by photosensitive agents. Veterinary Microbiology, v. 38, n. 3, p. 277-284, 1994.

7. EMILIO, C. R. Comparação da eficácia do ácido 5-aminolevuliníco com a de seu metil éster utilizando-se a terapia fotodinâmica no tratamento de carcinoma espinocelular felino. 2008. 127 p. Tese (Doutorado) - Instituto de Pesquisas Energéticas e Nucleares, São Paulo, 2008.

8. FELDMAN, B. C. Avaliação clínica dos efeitos da terapia fotodinâmica antimicrobiana no tratamento não-cirúrgico da periodontite crônica. 2009. 94 p. Dissertação (Mestrado) - Universidade do Grande Rio "Prof. José de Souza Herdy", Duque de Caxias, 2009.

9. HAGE, R.; MANCILHA, G.; ZANGARO, R. A.; MUNIN, E.; PLAPLER, H. Photodynamic Therapy (PDT) using intratumoral injection of the 5- aminolevulinic acid (5-ALA) for the treatment of eye cancer in cattle. Proceedings of SPIE, v. 6427, p. 642-671, 2007.

10. HAMBLIN, M. E.; HASAN, T. Photodynamic therapy: a new antimicrobial approach to infectious disease? Photochemistry and Photobiology Science, v. 3, n. 5, p. 436450, 2004

11. JORI, G.; MAGARAGGIA, M.; FABRIS, C.; SONCIN, M.; CAMERIN, M.; TALLANDINI, L.; COPPELLOTTI, O.; GUIDOLIN, L. Photodynamic inactivation of microbial pathogens: disinfection of water and prevention of water-borne diseases. Journal of Environmental Pathology, Toxicology and Oncology: Official Organ of the International Society for Environmental Toxicology and Cancer, v. 30, n. 3, p. 261271, 2011.

12. KESSEL, D. Photodynamic therapy: from the beginning. Photodiagnosis and Photodynamic Therapy, v. 1, n. 1, p. 3-7, 2004. 
13. KONOPKA, K.; GOSLINSKI, T. Photodynamic therapy in dentistry. Journal of Denta Research, v. 86, n. 11, p. 1126, 2007.

14. LUCROY, M. D. Photodynamic therapy for companion animals with cancer. The Veterinary Clinics of North America: Small Animal Practice, v. 32, n. 3, p. 693-702, 2002.

15. MARMUR, E. S.; SCHMULTS, C. D.; GOLDBERG, D. J. A review of laser and photodynamic therapy for the treatment of nonmelanoma skin cancer. Dermatologic Surgery, v. 30, pt. 2, p. 264-271, 2004.

16. MARTENS, A.; MOOR, A.; WAELKENS, E.; MERLEVEDE, W.; DE WITTE, P. In Vitro and In Vivo evaluation of hypericin for photodynamic therapy of equine sarcoids. The Veterinary Journal, v. 159, n. 1, p. 77-84, 2000.

17. MATEU, E.; MARTIN, M. Why is anti-microbial resistance a veterinary problem as well? Journal of Veterinary Medicine Series B-Infectious Diseases and Veterinary Public Health, v. 48, n. 8, p. 569-581, 2001.

18. OSAKI, T.; TAKAGI, S.; HOSHINO, Y.; AOKI, Y.; SUNDEN, Y.; OCHIAI, K.; OKUMURA $M$. Temporary regression of locally invasive polypoid rhinosinusitis in a dog after photodynamic therapy. Australian Veterinary Journal, v. 90, n. 11, p. 442-447, 2012.

19.PENG, Q.; MOAN, J.; NESLAND, J. M. Correlation of subcellular and intratumora photosensitizer localization with ultrastructural features after photodynamic therapy. Ultrastructural Pathology, v. 20, n. 2, p. 109-129, 1996

20.PIRES, L.; BOSCO, S. D. E. M.; DA SILVA JR., N. F.; KURACHI, C. Photodynamic therapy for pythiosis. Veterinary Dermatology, v. 24, n. 1, p. 130-136, 2013.

21. SELLERA, F. P.; FERNANDES, L.T.; POGLIANI, F. C.; TEIXEIRA, C. R.; DUTRA, G. H. P. LASSAVIA, C. N. Terapia fotodinâmica no tratamento de pododermatite em pinguinsde-Magalhães (Spheniscus magellanicus). In: CONGRESSO LATINO-AMERICANO DE REABILITAÇÃO DE FAUNA MARINHA, 2., 2012, Rio Grande. Anais... Rio Grande. 2012a. p. 51.

22. SELLERA, F. P.; FERNANDES, L. T.; POGLIANI, F. C.; TEIXEIRA, C. R.; DUTRA, G. H. P.; LASSAVIA, C. N. Terapia Fotodinâmica no Tratamento de Fibropapilomatose Cutânea em Tartarugas verdes (Chelonia mydas). In: CONGRESSO LATINO-AMERICANO DE REABILITAÇÃO DE FAUNA MARINHA, 2., 2012, Rio Grande. Anais... Rio Grande. $2012 b$

23. SELLERA, F. P.; GARGANO, R. G.; AZEDO, M. R.; BENESI, F. J.; ALMEIDA-LOPES L.; POGLIANI, F. C. Antimicrobial photodynamic therapy as an adjuvant treatment of toe ulcer in cattle. European International Journal of Science and Technology, v. 2, p. $98-104,2013 a$

24. SElleRA, F. P.; LASSAVIA, C. N.; AZEDO, M. R.; POGLIANI, F. C.; SELLERA D. P.; ARANHA, A. C. C. Photodynamic therapy in the treatment of canine oral papillomatosis. International Journal of Science Commerce and Humanities, v. 1, p. 23-27, 2013b.

25. SELLERA, F. P.; AZEDO, M. R.; SILVA, L. C. B. A.; SEINO, C. H.; GARGANO, R. G.; BATISTA, C. F.; DELLA LIBERA, A. M. M. P.; BENESI, F. J.; POGLIANI, F. C. Photodynamic therapy as a promising treatment of burn wounds after calf dehorning. International Journal of Science Commerce and Humanities, v. 1, p. 28-32, $2013 \mathrm{c}$.

26. SELLERA, F. P.; SOUZA, A. S. L.; FERREIRA, V. G.; ALVES, M. B. R.; AZEDO, M. R.; BENESI F. J.; POGLIANI, F. C. Photodynamic therapy in cattle hoof diseases. In: CONGRESSO MUNDIAL DE BUIATRIA, 27., 2012, Lisboa. Anais... Lisboa. 2012c. p. 267-268.

27. SILVIA, J. C.; LACAVA, Z. G.; KUCKELHAUS, S.; SILVA, L. P.; NETO, L. F.; SAURO, E. E.; TEDESCO, A. C. Evaluation of the use of low level laser and photosensitizer drugs in healing. Lasers in Surgery and Medicine, v. 34, n. 5, p. 451-457, 2004

28. TAKASAKI, A. A.; AOKI, A.; MIZUTANI, K.; SCHWARZ, F.; SCULEAN, A.; WANG, C. Y.; KOSHY, G.; ROMANOS, G.; ISHIKAWA, I.; IZUMI, Y. Application antimicrobial photodynamic therapy in periodontal and Peri-implant diseases. Periodontology 2000, v. 51, n. 1, p. 109-140, 2009.
29. USACHEVA, M. N.; TEICHERT, M. C.; BIEL, M. A. Comparison of the methylene blue and toluidine blue $\mathrm{O}$ bacterial efficacy against gram-positive and gram-negative microorganisms. Lasers in Surgery and Medicine, v. 29, n. 2, p. 165-173, 2001.

30.VILENSK, J.; KOUDINOVA, N. V.; HARMELIN, A.; SCHERZ, A.; SALOMON, Y Vascular-targeted photodynamic therapy (VTP) of a canine-transmissible venereal tumour in a murine model with Pd-bacteriopheophorbide (WST09). Veterinary and Comparative Oncology, v. 3, n. 4, p. 182-193, 2005.

31. WASHBURN, K. E.; STREETER, R. N.; SALIKI, J. T.; LEHENBAUER, T. W.; PRADO, M E. Photodynamic inactivation of an RNA enveloped virus in goat colostrum. Small Ruminant Research, v. 42, n. 1, p. 31-37, 2001.

32.WEIS, S. A.; DEN BERGH, H. V.; GRIFFIOEN, A. W.; NOWAK-SLIWINSKA, $P$. Angiogenesis inhibition for the improvement of photodynamic therapy: the revival of a promising idea. Biochimica et Biophysica Acta, v. 1826, n. 1, p. 53-70, 2012.

33. WYSS, P. History of photomedicine. In: WYSS, P.; TADIR, Y.; TROMBERG, B. J.; HALLER, U. Photomedicine in Gynecology and Reproduction. 2. ed. Switzerland: Karger Publishers, 2000. p. 4-11.

34. ZEITOUNI, N. C.; OSEROFF, A. R.; SHIEH, S. Photodynamic therapy for nonmelanoma skin cancers - current review and update. Molecular Immunology, v. 39, n. 17-18, p. $1133-1136,2003$

35.ZELICKSON, B. D. Mechanisms of action of topical aminolevulinic acid. In: GOLDMAN, M. P. (Ed.). Photodynamic Therapy. 1st ed. Philadelphia: Elsevier Saunders, 2005. p. 1-12. 